\title{
TEXTURAL THROUGH-THICKNESS INHOMOGENEITY AND ITS INFLUENCE ON THE PREDICTION OF PLASTIC ANISOTROPY
}

\author{
P. BLANDFORD, J.A. SZPUNAR, D. DANIEL and J. J. JONAS \\ Department of Metallurgical Engineering, McGill University \\ 3450 University Street, Montreal, Canada, H3A 2 A7.
}

Work done for this paper centres around two themes: 1) the characterization of the through-thickness inhomogeneity of texture observed in certain deep drawing steels, and 2) attempting to understand to what extent the presence of this inhomogeneity affects the correlation between the texture and the bulk plastic anisotropy of the specimens. For this, the textural inhomogeneities in three deep drawing steels, two interstitial free and one aluminum killed, were measured and described using the orientation distribution function (ODF). The bulk plastic anisotropies of these specimens were obtained through the measurement of the $r$-value. The impact of the measured inhomogeneities on the bulk properties was analyzed by comparing the r-value anisotropies calculated using a series expansion method from the various texture measurements made through the sheet thicknesses to the experimental $r$-values. It was observed that the textural inhomogeneities in the specimens were slight and consisted mainly of small Goss components at the surface and sub-surface of the sheets, which were not observed at the sheet centres. The impact of these inhomogeneities was small, but generally the $r$-value predictions made from the textures measured at the sheet centres were better correlated with the experimental $r$-value anisotropies.

\section{INTRODUCTION}

The through-thickness inhomogeneity of texture is often ignored and texture measurements made at the sheet centre are assumed to be representative of the bulk texture of the material. This is done even though it has been shown that textural inhomogeneities are present in specimens prepared both by rolling1-5 and annealing processes $2,6-8$.

For materials exhibiting texture gradients, it is not expected that the prediction of bulk material properties will be an easy task; in such cases, predictions could require a term characterizing the interaction between the differently textured layers. Such an interaction term could be quite 
complex, and the authors believe instead that the effect of the throughthickness inhomogeneity should be studied through practical experimentation?.

The results of such study could be used to recommend experimental methods for obtaining the optimal texture information for the purpose of accurate correlation.

\section{EXPERIMENTAL}

Three deep drawing steel specimens were studied, two of which were cut from continuous annealed, interstitial free steels (specimens IF1 and IF2), while the third was taken from a batch annealed, aluminum killed steel (specimen AKDQ). The final thicknesses for the specimens were 0.71 , 0.72 and $1.18 \mathrm{~mm}$, respectively.

The through-thickness inhomogeneity of the texture was measured by sequentially removing various amounts of material from the surface of the sheet and preparing that surface for texture measurement by polishing with 3 micrometer diamond paste, followed by electropolishing in a $60 \%$ $40 \%$ mixture of perchloric and glacial acetic acid. Reflection texture measurements were made on a Siemens texture system using a molybdenum X-ray source. The Siemens ODF/TEX11 software was used to calculate the coefficients of the ODF $\left(l_{\max }=22\right.$, no ghost correction) and plot the ODF sections, using three incomplete pole figures (the $\{110\},\{200\}$ and $\{211\})$.

$R$-value measurements were made at $15 \%$ elongation at $15^{\circ}$ intervals from the rolling direction.

\section{RESULTS AND ANALYSIS}

The texture measurements were made for each of the specimens at $S=1.0, S=0.95$ and $S=0.0\left(S=\left(t-t_{o} / 2\right) / t_{o} / 2\right.$, where $t_{0}$ is the original thickness of the specimen and $t$ is its thickness after removal of the surface).

These measurements showed that for all three specimens, the texture was similar and could be described as consisting of a well defined gamma fibre with some spread of orientation along the alpha fibre, making it convenient to depict the textural inhomogeneity using the $\Phi_{2}=45^{\circ}$ sections of the ODF. Figures 1a, b, and c show these sections for the textures measured at $S=1.0, S=0.95$ and $S=0.0$ for the first and second interstitial free steels (IF1 and IF2) and for the aluminum killed steel (AKDQ).

As shown in the $\Phi_{2}=45^{\circ}$ ODF sections in Figure 1a, there is a noticeable Goss component $(f(g) \simeq 1.7)$ at both $S=1.0$ and $S=0.95$ which is not present at $S=0.0$. It is accompanied by a general increase in the value of the orientation densities observed along the gamma fibre from the surface of the sheet towards the sheet centre. All three textures measured have higher $\{111\}<110>$ orientation densities than $\{111\}<112>$ densities.

This is in contrast to the textures measured at various layers in the second interstitial free steel specimen (IF2, Figure 1b). In this case, the 


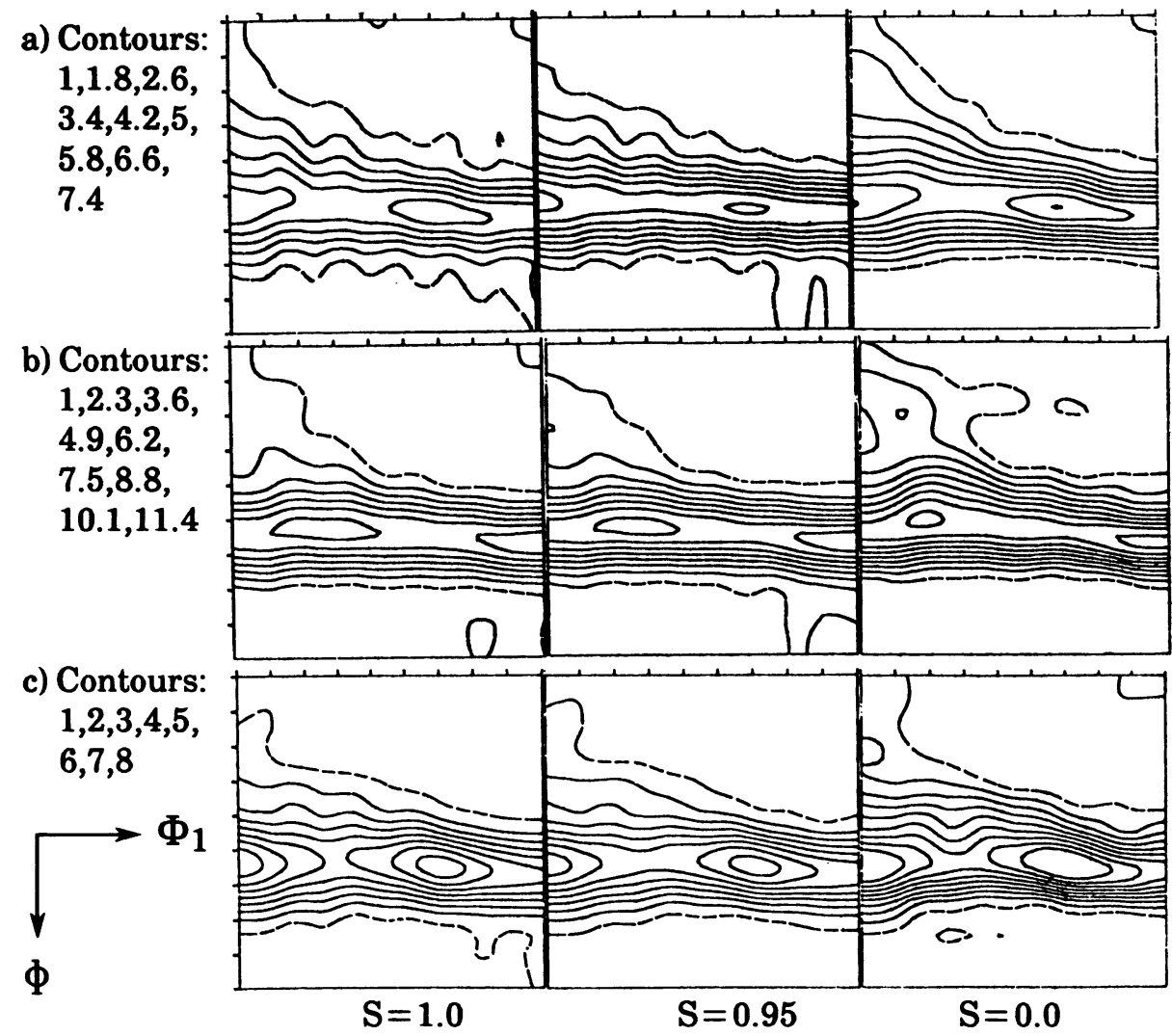

Figure 1: $\phi_{2}=45^{\circ}$ ODF sections for steel a) IF1, b) IF2, and 3) AKDQ.

trend is for the $\{111\}<112>$ orientation to be stronger than the $\{111\}<110\rangle$ orientation. As in the previous interstitial free specimen, the strength of texture along the gamma fibre increases as we move from the sheet surface towards the sheet centre. Also, there is some Goss component observed at the sheet surface and sub-surface which is not observed at the sheet centre.

Figure 1c, containing the $\Phi_{2}=45^{\circ}$ ODF sections for the aluminum killed steel specimen, shows traces of Goss texture at $S=1.0$ which is not observed at the sub-surface level of $S=0.95$. As in the case of the previous two deep drawing steels, the average strength of the texture along the gamma fibre increases from the sheet surface towards the sheet centre, with stronger textures observed at the $\{111\}<112>$ orientations than at the $\{111\}<110>$ orientations.

To investigate the effect that these textural inhomogeneities have on the correlation of texture with the $r$-value anisotropy, the experimentally measured r-values ${ }^{10}$ were compared with theoretical $r$-values calculated for each of the textures measured through the sheet thickness. For each steel, 
that combination of plasticity model and slip system 10 was chosen which minimized the difference between the experimental $r$-value anisotropy and the anisotropy predicted using the straight average of the ODF coefficients obtained from the textures measured at $S=1.0, S=0.95$ and $S=0.0$. In this fashion the following plasticity models and slip systems were chosen:

1) a Taylor or full constraints model with a critical resolved shear stress (CRSS) ratio between competing $\{111\}<112>$ and $\{111\}<110>$ slip systems equal to 0.95 (i.e. $\tau\{112\} / \tau\{110\}=0.95$ ) for the first interstitial free steel specimen (IF1);

2) a 'pancaked-grain', relaxed constraints model $\left(\varepsilon_{13}\right.$ and $\varepsilon_{23}$ strains relaxed) with $\tau\{112\} / \tau\{110\}=0.95$ for the second interstitial free steel specimen (IF2); and

3) a 'pancaked-grain', relaxed constraints model with $\tau\{112\} / \tau\{110\}=0.9$ for the aluminum killed steel specimen.

The results obtained using this selection process are presented in Figures 2a, b, and c.

For the first interstitial free steel specimen, a comparison of the $r$ value anisotropies predicted from the textures measured at the various layers through the sheet thickness and the experimental $r$-value (Figure 2a)

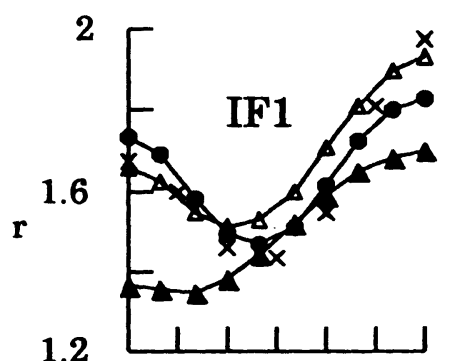

a)

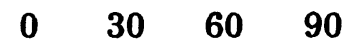

$\beta\left(^{\circ}\right)$

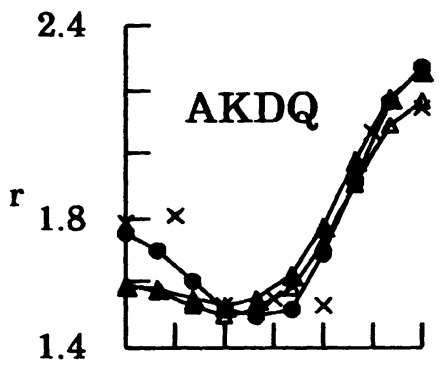

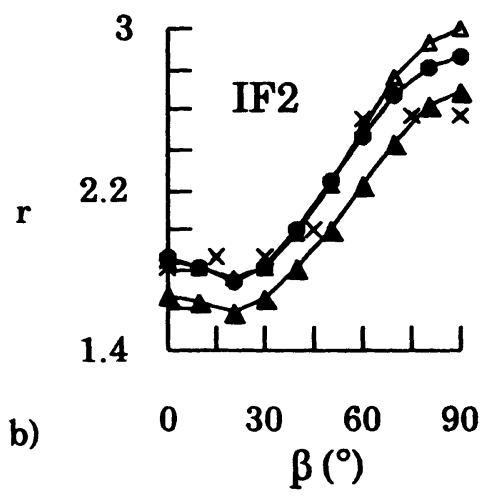

Legend:

$\begin{array}{ll}\mathrm{S}=1.0 & \Delta \\ \mathrm{S}=0.95 & \Delta \\ \mathrm{S}=0.0 & \bullet \\ \mathrm{EXP} . & \mathrm{x}\end{array}$

c) $\quad \begin{array}{llll}0 & 30 & 60 & 90\end{array}$

Figure 2: Comparison of the predicted and experimental $r$-values. 
shows that the prediction based on the texture measured at the sheet centre and at the sheet sub-surface (i.e. $S=0.95$ ) is quite good; in contrast, the prediction obtained from the surface texture does not agree so well, especially for those directions close to the rolling direction.

Figure 2b shows the results for the second IF steel specimen. In this case, again, the predictions from the textures measured at the sub-surface and the sheet centre are similar, and for the most part they best fit to the experimental $r$-value data. The prediction from the surface texture is noticeably different from those predicted from the other layers, although the difference is not so much exhibited by a change in the variation of $\mathbf{r}$ as by a change in the average value.

Lastly, a comparison of the predicted and experimental $r$-value anisotropy for the aluminum killed steel specimen (Figure 2c) shows that the prediction using the ODF coefficients obtained from the texture at the sheet centre fits better than the predictions from the other texture measurements. The predictions using the coefficients of the sheet surface and sub-surface texture are similar to each other and diverge from the experimental r-value anisotropy, especially for directions close to the rolling direction.

\section{DISCUSSION AND CONCLUSIONS}

The texture measurements made on each of the three deep drawing steels indicate that these steels have very little through-thickness inhomogeneity. Any that exists is mostly concentrated within the surface 5$10 \%$ of the sheet thickness where the texture contains a small Goss component which is not observed in the rest of the specimen. The texture of the bulk was noticeably different for each of the three steels. A comparison of the gamma fibres of these steels showed that both the AKDQ and the IF1 steels had a similar texture, favouring the $\{111\}<110>$ component over the $\{111\}<112>$ component, but the extent to which the $\{111\}<110>$ component predominated was greater in the AKDQ steel. On the other hand, the gamma fibre of the bulk texture of the IF2 steel was generally more even, with no particular component predominating.

For each steel, the effect of the measured inhomogeneity on the $r$ value anisotropy was studied by choosing the plasticity model and CRSS ratio which produced the best fit with the experimentally measured $r$ values. The optimal model for the aluminum-killed steel, AKDQ, was found to be a relaxed constraints model with the $\varepsilon_{13}$ and the $\varepsilon_{23}$ strains relaxed, using a CRSS ratio for the $\{112\}<111>$ and $\{110\}<111>$ slip systems equal to $0.9\left(\tau_{\{112\}} \tau_{\{110\}}=0.9\right)$. A Taylor, full constraints model using mixed slip, $\tau_{\{112\} /} \tau_{\{110\}}=0.95$, provided the optimal fit for the first interstitial-free steel, IF1, while for the second interstitial-free steel (IF2), the same relaxed constraints model as that used for the AKDQ steel provided the best fit with the experimental r-value anisotropy by using a CRSS ratio of $\tau_{\{112\}} \backslash\{110\}=1.0$. 
The selection of the 'pancaked grain' plasticity model for these steels is in good agreement with the results obtained on the same steels by Daniel and Jonas 10. The variation in the optimal CRSS ratio found as a result of selecting the best fit with the experimental $r$-value anisotropy was explained in this work by the variation in chemical composition between the steels. Support for this can be found in the variation of the CRSS ratio determined in single crystal work by Franciosi7 and by Orlans-Joliet, et al11.

Having chosen the optimal plasticity model in this way, it was found that the effect that the textural inhomogeneity has on the r-value anisotropy is slight. Conclusively for the AKDQ and IF1 steels, the predictions obtained using the texture of the bulk (i.e. the texture measured at $\mathbf{S}=0.0$, which from the analysis is the texture of the bulk) are very close to the measured values while the predictions from the surface texture have noticeably different shapes. From the predictions made from the measurements of the IF2 steel, there is some indication that predictions from the bulk texture are more accurate. The significance of this conclusion is that for correlating texture and plastic properties of cold-rolled sheet exhibiting little through-thickness inhomogeneity, the bulk texture must be measured and used.

AKNOWLEDGMENTS: The authors would like to thank the National Science and Engineering Research Council and the Ministry of Education of Quebec (FCAR) for the funding of this work, and the Kawasaki Steel Corporation of Japan for providing the steels and r-value measurements. $P$. Blandford would like to thank Stelco Inc. for his Stelco Research Scholarship.

\section{REFERENCES}

1 P.I. Welch, H.J. Bunge and C.M. Vlad, Arch. Eisenhuttenwes, 55, No.7, 321 (1984).

2 S. Mishra, C. Darmann and K. Lucke, Acta metall., 32, 2185 (1984).

$3 \quad$ M. Matsuo,T. Sakai and Y. Suga, Metall. Trans., 17A, 1313 (1986).

4 C.A. Stickels, Transactions of the Metallurgical Society of the AIME, 239, 1857 (1967).

5 W. Truszkowski, J. Krol and B. Major, Proc. of the $8^{\text {th }}$ Int. Conf. on Textures of Materials, (AIME, Warrendale 1988), p. 39.

6 J.H. Held, Transactions of the Metallurgical Society of the AIME, 239, 573 (1967).

$7 \quad$ P. Franciosi, Acta metall., 31, 1331 (1983).

8 J. Pospiech, and J. Jura, Z. Metallkunde, 65, 324 (1974).

9 P. Blandford and J.A. Szpunar, IEEE Trans. on Magnetics, to be published (1990).

10 D. Daniel and J.J. Jonas, Metall. Trans., 21 A, 331 (1990).

11 B. Orlans-Joliet, J.H. Driver and F. Montheillet, Acta metall., 38, 581 (1990). 